\title{
Prevalencia de ansiedad y depresión en pacientes de hemodiálisis
}

\author{
Lídia Gómez Vilaseca, Nuria Pac Gracia, Mónica Manresa Traguany, Sandra Lozano Ramírez, Julio Leonel \\ Chevarria Montesinos
}

Servicio de Nefrología, Hospital de Palamós. Gerona. España

\section{Resumen}

Introducción: Los pacientes en hemodiálisis tienen síntomas y trastornos emocionales como ansiedad y depresión. Son pocos los estudios que valoren el diagnóstico mediante la Hospital Anxiety and Depression Scale (HADS); nuestro objetivo es conocer la prevalencia de la ansiedad y depresión en pacientes con enfermedad renal crónica en programa de hemodiálisis.

Metodología: Estudio transversal durante el primer trimestre del 2012. Realizado en el servicio de hemodiálisis del hospital de Palamós. Se incluyeron pacientes en programa crónico de hemodiálisis que llevaban como mínimo un mes. Se registró la edad, sexo, talla, peso, índice de masa corporal, índice de Charlson, tiempo en hemodiálisis y número de fármacos. Se utilizó la escala HADS (versión española de Caro-Ibáñez).

Resultados: Se analizaron 49 pacientes, $25 \%$ fueron mujeres, la edad media 67,2 años, I. Charlson 4,6 ( DE:4,5), tiempo en HD 39,9 meses (DE:43,8), IMC 26,9 (DE:4,5), turno de mañana $52,9 \%$ y tarde 50,9 $\%$. La sintomatología depresiva representa $42,9 \%$ (IC95\% 33,7\%-60,6\%) y la ansiosa $32,7 \%$ (IC95\% $21,2 \%-46,6 \%$ ) según la escala HADS. La ansiedad presenta relación estadísticamente significativa con el índice de masas corporal inferior y sexo femenino, la depresión con una edad más elevada, índice de masa corporal inferior y el turno de la mañana.

Conclusiones: Existe una alta prevalencia de ansiedad y depresión en pacientes con enfermedad renal crónica en hemodiálisis. Un índice de masa corporal bajo se relaciona con la ansiedad y depresión, la mayor edad con la depresión y la ansiedad es más frecuente en muje-

Correspondencia:
Lídia Gómez Vilaseca
C/. Carmel, ${ }^{\circ} 44$ bajos
17230 Palamós. Gerona
E-mail: lidiagomezvilaseca@gmail.com

res. Nuestro estudio sugiere que es necesario un mayor control, seguimiento y tratamiento de las alteraciones emocionales en pacientes con enfermedad renal crónica.

PALABRAS CLAVE

- DEPRESIÓN

- ANSIEDAD

- HEMODIÁLISIS

- ESCALA HADS

- DIAGNÓSTICO

Prevalence of anxiety and depression in hemodialysis patients

\begin{abstract}
Introduction: Hemodialysis patients have symptoms and emotional disorders such as anxiety and depression. There are few studies that evaluate diagnosis using the Hospital Anxiety and Depression Scale (HADS); our aim is to determine the prevalence of anxiety and depression in patients with chronic kidney disease on hemodialysis.
\end{abstract}

Methods: Cross-sectional study during the first quarter of 2012 was carried out. It was conducted in the hemodialysis service of the Palamós Hospital. Patients on chronic hemodialysis with a dialysis vintage at least than a month were included. Age, sex, height, weight, body mass index, Charlson index, time on hemodialysis and number of drugs were recorded. HADS (Spanish version of Caro-Ibanez) scale was used.

Results: 49 patients were analyzed, $25 \%$ being women. The mean age was 67.2 years, the average Charlson index was 4.6 (SD: 4.5), a dialysis vintage of 39.9 
months (SD: 43.8) and an average BMI of 26.9 (SD: 4.5). The patients in the morning shift were $52.9 \%$ and in the evening shift were $50.9 \%$. Depressive symptomatology was represented in $42.9 \%$ (95\% CI $33.7 \%$ $-60.6 \%)$ and anxiety symptoms in $32.7 \%(95 \% \mathrm{CI}$ $21.2 \%-46.6 \%$ ) according to HADS scale. The anxiety has significant association with lower body mass index and female, while depression is significantly associated with higher age, lower body mass index and the morning shift.

Conclusion: There is a high prevalence of anxiety and depression in patients with chronic kidney disease on hemodialysis. A low body mass index is associated with anxiety and depression, older age with depression, and anxiety is more common in women. Our study suggests that greater control, monitoring and treatment of emotional disorders in patients with chronic kidney disease are necessary.

\section{KEYWORDS}

- DEPRESSION

- ANXIETY

- HEMODIALYSIS

- HADS SCALE

- DIAGNOSIS

\section{Introducción}

Existe una alta prevalencia de ansiedad y depresión en pacientes con enfermedad renal crónica terminal, la depresión aparece como la segunda condición de comorbilidad en pacientes con insuficiencia renal grave que requieren hemodiálisis (HD)1,2; además se ha descrito que una cuarta parte de los pacientes tenían ansiedad y la otra mitad síntomas cognitivos de depresión ${ }^{3}$. Algunos de los síntomas asociados a la ansiedad y depresión son confundidos con los de la enfermedad renal crónica (ERC), muchos de estos no son específicos y en general son fluctuantes, condicionando de forma significativa el bienestar general, siendo a veces difícil determinar si existe una causa orgánica ${ }^{4,5}$.

Varias investigaciones enfatizan que la depresión en pacientes renales crónicos se encuentra asociada a comportamientos de no adhesión al tratamiento médico, lo cual conduce a una deterioro de la calidad de vida, a una salud más pobre y un aumento de los riesgos de mortalidad ${ }^{1,6,7}$.
La prevalencia de estos trastornos difiere según los diversos autores entre el 25 y el $50 \%$ y algunos llegan hasta el $70 \%$. Los factores que explican tanta variabilidad pueden ser las diferencias en el proceso de selección de los pacientes y los métodos de cribado y diagnóstico utilizados ${ }^{8}$.

La intensidad de las alteraciones emocionales, varía de un paciente a otro dependiendo de la valoración cognitiva que se realice del impacto de la enfermedad renal y la HD sobre sus condiciones de vida; también se relaciona con las habilidades individuales para adaptarse a la condición de la dependencia del programa de HD.

En el estudio de Amelia E. Páez, que utilizó la escala de Depresión de Beck-II y el inventario de ansiedad Rasgo-Estado de Spielberger, encontraron que el $56,7 \%$ manifestó algún grado de depresión, relacionando positivamente los niveles de ansiedad (estadorasgo) con los niveles de depresión?

La prevalencia de síntomas ansiosos detectada en el trabajo de Moreno y cols, en Alicante, es del $46,6 \%$, con un mayor porcentaje para ansiosos leves $(33,3 \%)$, porcen-taje similar o levemente superior al hallado en otros estudios ${ }^{8}$.

En el trabajo de Muñoz y Cols, demuestra que en pacientes de hemodiálisis un $25 \%$ presenta depresión y $17 \%$ ansiedad, utilizando la escala HADS $^{10}$.

Coloma y Cols, muestran que el $54,3 \%$ de los pacientes tienen depresión en algún grado y tan solo el $27.6 \%$ tienen ansiedad moderada o severa, en su estudio en Mála$\mathrm{ga}^{11}$.

Alvarez-Ude y Cols, obtuvieron la existencia de sintomatología depresiva relevante (moderada/severa) en $43 \%$, $24 \%$ tenían Ansiedad estado y $31 \%$ Ansiedad Rasgo 4 .

Kurella y Cols en el 2005, concluyeron que la depresión en pacientes renales aumenta en un $84 \%$ las probabilidades de suicidio en comparación con la población general ${ }^{12}$.

García Llana y Cols ${ }^{13}$, en el 2014 publicaron una revisión donde encontraron que un 35\% de los estudios revisados utilizan el instrumento HADS para evaluar la ansiedad y depresión de los 38 estudios revisados. En las 16 muestras la prevalencia de la de-presión se sitúa entre el $25,8 \%$ y el $68,1 \%$. Un estudio encontró una prevalencia de $71,4 \%$ de trastornos psiquiátricos medida a través de la entrevista semiestructurada basada en el diagnostic and statistical manual of Mental disorders (DSM)-IV, de los cuales un $20 \%$ correspon- 
día a una depresión mayor y un 10\% a distimia, únicamente tres informan de la prevalencia de ansiedad, situándose entre el $21 \%$ al $35,3 \%$.

La escala HADS se ha validado previamente en varios estudios, la revisión de The validity of the Hospital Anxiety and Depression Scale. An updated literatura review, analizó 747 artículos, y se concluye que esta escala es un buen indicador para evaluar sintomatología ansiosa i depresiva en pacientes de población general, pacientes de atención primaria y pacientes con patología crónica y psiquiátrica ${ }^{14}$.

Dada la relevancia de un pronto y adecuado diagnóstico que permita hacer el tratamiento y seguimiento de estos casos, y que existen muy pocos estudios que hayan valorado la ansiedad y depresión mediante el cuestionario Hospital Anxiety-Depression (HADS), nuestro objetivo es conocer la prevalencia de la ansiedad y depresión en pacientes con ERC en programa de hemodiálisis crónica mediante el cuestionario HADS.

\section{Metodología}

Estudio descriptivo observacional de corte transversal, durante el primer trimestre de 2012, en el servicio de diálisis del Hospital de Palamós. Se incluyeron un total de 56 pacientes en programa crónico de diálisis, que llevaban como mínimo un mes de tratamiento, mayores de 18 años y que aceptaron participar en el estudio. Se excluyeron del estudio 7 pacientes por patología psiquiátrica, incapacidad para responder el cuestionario y dificultad idiomática. El cuestionario se respondió durante la sesión de diálisis, por el mismo paciente o con ayuda.

El estado de ansiedad y/o depresión se evaluó mediante el cuestionario Hospital Anxiety-Depression (HADS) $)^{15,16}$. Cuestionario auto-administrado con 7 ítems referidos a la sub-escala de depresión y 7 referidos a la sub-escala de ansiedad. Cada ídem adopta un valor de 0 a 3 en una escala de tipo Likert de forma que la mínima y la máxima puntuación para cada subescala es de 0 y 21 respectivamente. Un valor inferior a 7 es ausencia de trastorno; los valores entre 8 y 10 son resultados dudosos y los valores de 11 o superiores indican presencia de ansiedad o depresión. Se elaboró un registro para la edad, sexo, talla, peso, índice de masa corporal (IMC), índice de Charlson, tiempo en hemodiálisis en meses, numero de fármacos que tenían prescritos, además se incluyeron dos preguntas para conocer la opinión del paciente respecto el soporte social y el grado de satisfacción de este.
El análisis estadístico de datos univariante se realizó mediante frecuencias, porcentaje, media y desviación estándar. El análisis inferencial de datos categóricos se realizó mediante los test de Ji2 o de Fisher. La normalidad de las variables continúas se evaluó mediante el test Shapiro-Wilks, y posteriormente la significación estadística mediante los test T-student. El riesgo estimado se valorará mediante el odds ratio $(\mathrm{OR})$ con un intervalo de confianza del $95 \%$. El análisis se hizo con el programa estadístico SPSS19.

\section{Resultados}

Se analizaron un total de 49 pacientes, los datos descriptivos generales se aprecian en la Tabla 1.

Tabla 1. Características de los pacientes $(n=49)$.

\begin{tabular}{ll}
\hline Características & Media (DE) \\
\hline Sexo $(\mathrm{D} / \mathrm{H}) \%$ & $14 / 35(28.6 / 71.4)$ \\
\hline Talla $(\mathrm{cm})$ & $164(9,3)$ \\
\hline Peso $(\mathrm{kg})$ & $73,1(15,4)$ \\
\hline Edad (años) & $67,2(16,2)$ \\
\hline Índice de Charlson & $4,7(1,8)$ \\
\hline Índice de masa corporal $\left(\mathrm{Kg} / \mathrm{m}^{2}\right)$ & $26,9(4,5)$ \\
\hline Tiempo en Hemodiálisis $(\mathrm{meses})$ & $39,9(43,8)$ \\
\hline No de Fármacos & $10,9(2,7)$ \\
\hline
\end{tabular}

En nuestro estudio hemos encontrado que las mujeres presentan mayor ansiedad, además también tienen mayor prevalencia de depresión, aunque no es significativa.

Respecto al cuestionario HADS para valorar la ansiedad (Gráfico 1), se observaron la ausencia de trastorno en un $67,3 \%$, un resultado dudoso en $20,4 \%$ y con trastorno de ansiedad definido $12,2 \%$.

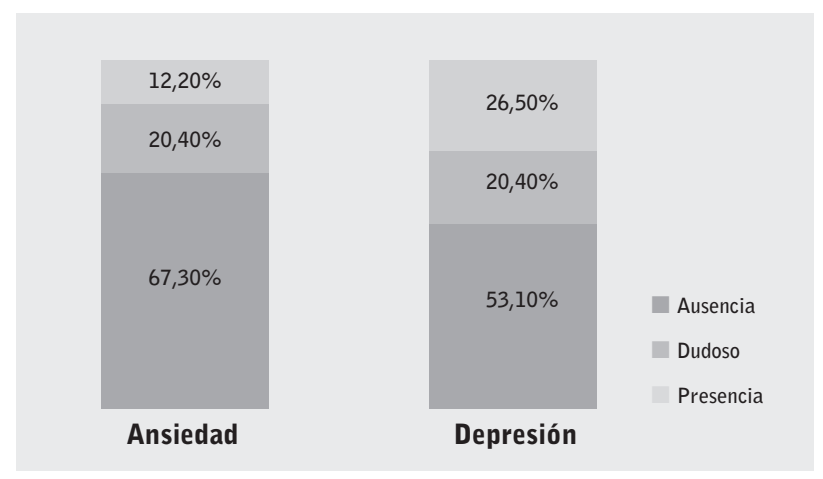

Gráfico 1. Prevalencia de ansiedad y depresión. 
Respecto a la depresión (Gráfico 1), se observaron la ausencia de trastorno en $53,1 \%$, un resultado dudoso en $20,4 \%$ y con trastorno de depresión $26,5 \%$.

Un $53,1 \%$ respondió que contaba con más de dos personas de ayuda, $20,4 \%$ dos personas, $24,5 \%$ una persona y $2 \%$ ninguna. El $63,3 \%$ considera muy satisfac- toria la ayuda que reciben, $28,6 \%$ satisfactoria, $4,1 \%$ solo un poco y $4,1 \%$ deficiente.

La relación entre la presencia o ausencia de trastorno con las variables que consideramos para el estudio se muestran en la Tabla 2.

Tabla 2. Sintomatología ansiosa y depresiva en función de la edad, índice de Charlson, IMC y tiempo en HD.

\begin{tabular}{|c|c|c|c|c|c|c|c|c|}
\hline & Ansiedad & $\mathbf{N}$ & Media & $\mathbf{p}$ & Depresión & $\mathbf{N}$ & Media & $\mathbf{p}$ \\
\hline \multirow[t]{2}{*}{ Edad } & Ausencia & 33 & 65,3 & 0,33 & Ausencia & 26 & 58,1 & 0,01 \\
\hline & Ansiedad & 16 & 71,1 & & Depresión & 23 & 77,5 & \\
\hline \multirow[t]{2}{*}{ I. Charlson } & Ausencia & 33 & 4,4 & 0,29 & Ausencia & 26 & 4,5 & 0,49 \\
\hline & Ansiedad & 16 & 5,2 & & Depresión & 23 & 4,9 & \\
\hline \multirow[t]{2}{*}{ IMC } & Ausencia & 30 & 27,8 & 0,01 & Ausencia & 24 & 28,6 & 0,008 \\
\hline & Ansiedad & 16 & 25 & & Depresión & 22 & 25 & \\
\hline \multirow[t]{2}{*}{ Tiempo HD } & Ausencia & 32 & 38,8 & 0,87 & Ausencia & 26 & 43,5 & 0,56 \\
\hline & Ansiedad & 15 & 42,3 & & Depresión & 23 & 35,9 & \\
\hline S. Femenino / & Ausencia & $6 / 27$ & $42.9 / 77.1 \%$ & 0,02 & Ausencia & $5 / 21$ & $35.7 / 60 \%$ & 0,1 \\
\hline S. Masculino & Ansiedad & $8 / 8$ & $57.1 / 22.8 \%$ & & Depresión & $9 / 14$ & $64.3 / 40 \%$ & \\
\hline
\end{tabular}

*Para el turno de HD la p=0,82 y 0,026 ansiedad y depresión respectivamente.

Se observa que existe una relación estadísticamente significativa entre la presencia de ansiedad y el IMC, sexo, no así para la edad, I. Charlson, tiempo en HD y el turno de HD.

EI IMC es menor en los pacientes que presentan ansiedad comparado con los que no tienen ansiedad y la proporción de mujeres que presenta ansiedad es mayor.

En los pacientes con depresión se observa que tienen una edad media más elevada en comparación con los que no padecen ( 77,5 vs 58,1 años), el índice de masa corporal de estos pacientes es inferior, siendo estadísticamente significativas, además del turno de HD. No existe relación con el I. de Charson, tiempo en HD y el sexo.

El turno donde se detectaron niveles de depresión más altos fue el turno del martes-jueves-sábado por la mañana con una prevalencia de depresión del 40,9\%.

\section{Discusión}

Hemos observado que existe una mayor frecuencia de varones en nuestro servicio, además la edad media es semejante a la media nacional. Existe un alto grado de comorbilidad en general y la mayor parte de nuestra muestra se encuentra con peso normal o sobrepeso leve.

El cumplimiento del test, no requiere mucho tiempo y es de fácil comprensión. Según Zigmond y cols la escala puede ser auto-administrada o administrada por el evaluador ${ }^{15}$.

El cuestionario HADS, excluye en sus respuestas la posible patología física que muchas veces se somatiza y engloba la cognición y la psique en los resultados, haciendo que estos sean más específicos. Hemos decidido englobar los resultados dudosos (puntaje $\geq 8$ ) y los resultados significativos en una misma esfera, ya que pensamos que estos ya podrían padecer ya algún grado de patología (ansiedad y depresión) que tendría que ser valorada. La prevalencia de ansiedad en nuestro centro es de $32,6 \%$ (IC 95\%: $21,2 \%-46,6 \%$ ). Al igual que en la ansiedad donde la prevalencia final de 
depresión es del 46,9\%. (IC 95\%: 33,7\%-60,61\%), situándose en el rango mencionado en la revisión de García-Llana y otros autores ${ }^{8,9,13}$, que va del 10 al $50 \%$.

Como manifiestan otros autores la edad de los pacientes determina el comportamiento de la funcionalidad física y de su cualidad de vida; describiéndose una correlación entre la edad y la depresión ${ }^{17}$.

En nuestro estudio el IMC es elevado, se han descrito una menor mortalidad en los pacientes de HD que presentan un IMC ligeramente elevado, lo cual puede estar en relación a una mejor calidad de vida y por tanto menores índices de depresión y ansiedad ${ }^{18,19}$.

El turno donde presentaron niveles más altos de depresión es el turno de las mañanas (primeras horas de la mañana), aunque es posible que en este turno coinciden los pacientes más añosos y con un índice de comorbilidad más alto, debido a que en nuestro centro hospitalario la mayor demanda de servicios se realiza por las mañanas.

El porcentaje de pacientes que tienen aislamiento social es bajo, la gran mayoría tiene un buen soporte y refieren que es muy satisfactorio.

Las limitaciones que podemos señalar, es que hemos usado solamente el test HADS para valorar estos trastornos, sin embargo sería recomendable contar con mayor apoyo clínico y/o de otros test también validados que mejoren la precisión diagnóstica.

Como conclusión podemos decir que existe una alta prevalencia de ansiedad y depresión en la muestra estudiada, en un grado tal que precisaría algún tipo de intervención o tratamiento, ya que la importante repercusión que los trastornos emocionales tienen sobre el bienestar de los pacientes, así como su posible influencia sobre la supervivencia, hacen imprescindible su abordaje diagnóstico y terapéutico.

\section{Agradecimientos}

Agradecemos al equipo del Servicio de Nefrología del Hospital de Palamós, a la Psicóloga Monserrat Planas por su colaboración en la realización de este trabajo.

El presente trabajo se ha presentado como trabajo de investigación final del Máster de Psicología Clínica de la Universidad de Girona.

\section{Recibido: 20 diciembre 2014 \\ Revisado: 10 enero 2015 \\ Modificado: 15 febrero 2015 \\ Aceptado: 20 febrero 2015}

\section{Bibliografía}

1. Peterson RA, Kimmel PL, Sacks CR, Mesquita $M L$, Simmens SJ, Reiss D. Depression, perception of illness and mortality in patients with endstage renal disease. Int $\mathrm{J}$ Psychiatry Med. 1991;21(4):343-54.

2. Burton HJ, Kline SA, Lindsay RM, Heidenheim AP. The relationship of depression to survival in chronic renal failure. Psychosom Med. 1986 Apr;48(34):261-9.

3. Álvarez-Ude F, Fernández-Reyes MJ, Vázquez $A$, Mon C, Sánchez R, Rebollo P. Síntomas físicos y trastornos emocionales en los pacientes en programa de hemodiálisis periódica. Nefrol Publ of Soc Esp Nefrol. 2001 Apr;21(2):191-9.

4. Álvarez-Ude F, Galán P. Adaptación transcultural y validación preliminar de la versión española del Kidney Disease Questionnaire (Cuestionario de la enfermedad renal). Nefrol Publ Of Soc Esp Nefrol. 1997;17(6):486-96.

5. Parfrey PS, Vavasour HM, Henry S, Bullock $\mathrm{M}$, Gault MH. Clinical features and severity of nonspecific symptoms in dialysis patients. Nephron. 1988;50(2):121-8.

6. Kimmel PL. Psychosocial factors in adult end-stage renal disease patients treated with hemodialysis: correlates and outcomes. Am J Kidney Dis Off J Natl Kidney Found. 2000 Apr;35(4 Suppl 1):S132-40.

7. Untas A, Aguirrezabal M, Chauveau P, Leguen $E$, Combe C, Rascle N. [Anxiety and depression in hemodialysis: validation of the Hospital Anxiety and Depression Scale (HADS)]. Néphrologie Thérapeutique. 2009 Jun;5(3):193-200.

8. Moreno Núñez $E$, Arenas Jiménez MD, Porta Bellmar E, Escalant Calpena L, Cantó García MJ, Castell García G, et al. Estudio de la prevalencia de trastornos ansiosos y depresivos en pacientes en hemodiálisis. Rev Soc Esp Enferm Nefrológica. 2004 Dec;7(4):17-25. 
9. Páez A, Jofré M, Bortoli MÁ de. Ansiedad y depresión en pacientes con insuficiencia renal crónica en tratamiento de diálisis. Univ Psychol. 2009 May 27;8(1):117-24.

10. Muñoz Sancho R, Oto Royo A, Barrio Alonso R, Fernández M. Evolución de la calidad de vida en pacientes en hemodiálisis: Estudio prospectivo a un año. Rev Soc Esp Enferm Nefrológica. 2006 Mar;9(1):55-8.

11. Amador R, Pons E, Espinosa C. Depresión y ansiedad en pacientes en hemodiálisis: la creatividad para combatirlas. Comunicaciones Presentadas al XXXIV Congreso Nacional SEDEN; 2009.

12. Kurella M, Kimmel PL, Young BS, Chertow GM. Suicide in the United States end-stage renal disease program. J Am Soc Nephrol JASN. 2005 Mar;16(3):774-81.

13. García-Llana H, Remor E, Del Peso G, Selgas R. The role of depression, anxiety, stress and adherence to treatment in dialysis patients' health-related quality of life: a systematic review of the literature. Nefrol Publ Of Soc Esp Nefrol. 2014 Sep 26;34(5):63757.

14. Bjelland I, Dahl AA, Haug TT, Neckelmann D. The validity of the Hospital Anxiety and Depression Scale. An updated literature review. J Psychosom Res. 2002 Feb;52(2):69-77.
15. Zigmond AS, Snaith RP. The hospital anxiety and depression scale. Acta Psychiatr Scand. 1983 Jun;67(6):361-70.

16. Soto J. Las emociones como variables relacionadas con el cambio de hábitos de salud en familiares y amigos próximos de enfermos de cáncer. PSICOONCOLOGÍA. 2003;0(1):75-82.

17. Mirowsky J, Ross CE. Age and depression. J Health Soc Behav. 1992 Sep;33(3):187-205; discussion 206-12.

18. Zhao G, Ford ES, Dhingra S, Li C, Strine TW, Mokdad AH. Depression and anxiety among US adults: associations with body mass index. Int $\mathrm{J}$ Obes 2005. 2009 Feb;33(2):257-66.

19. Revah-Levy A, Speranza M, Barry C, Hassler C, Gasquet I, Moro M-R, et al. Association between Body Mass Index and depression: the. BMC Public Health. 2011 Aug 16;11(1):649.

20. Barrett BJ, Vavasour HM, Major A, Parfrey PS. Clinical and psychological correlates of somatic symptoms in patients on dialysis. Nephron. 1990;55(1):10-5.

21. Fishbein LJ. Depression in End-Stage Renal Disease Patients. Semin Dial. 1994;7(3):181-5. 